\title{
Study of a Linear Acoustooptic Laser Modulator Based on All-Fibre Sagnac Interferometer
}

\author{
G. Trinidad García, ${ }^{1}$ E. Molina Flores, ${ }^{2}$ B. A. Ramírez Solís, ${ }^{2}$ and H. Azucena Coyotecatl ${ }^{2}$ \\ ${ }^{1}$ Facultad de Ciencias Físico Matemáticas, Posgrado en Física Aplicada, Benemérita Universidad Autónoma de Puebla, \\ Boulevard 18 Sur y Avenida San Claudio, San Manuel, 72570 Puebla, PUE, Mexico \\ ${ }^{2}$ Facultad de Ciencias de la Electrónica, Benemérita Universidad Autónoma de Puebla, Boulevard 18 Sur y Avenida San Claudio, \\ San Manuel, 72570 Puebla, PUE, Mexico
}

Correspondence should be addressed to E. Molina Flores; emolina@ece.buap.mx

Received 9 February 2016; Revised 11 May 2016; Accepted 24 May 2016

Academic Editor: Somenath N. Sarkar

Copyright ( 2016 G. Trinidad García et al. This is an open access article distributed under the Creative Commons Attribution License, which permits unrestricted use, distribution, and reproduction in any medium, provided the original work is properly cited.

\begin{abstract}
The feasibility of polarization-maintaining photonic crystal fibre (PM-PCF) strategy for acoustooptic modulation using all-fibre Sagnac interferometer is demonstrated. The principal constraint to apply the strategy is defined by a linear laser acoustooptic modulator $(\mathrm{AOM})$ for $1550 \mathrm{~nm}$. The intensity of incident acoustic waves over the PM-PCF loop segment affected the signal interference transmission; here, modulation by birefringence variation around $7.6 \times 10^{-4} \pm \Delta n_{i}$ was observed. It is discovered that, through mathematical analysis, two operation points in the spectrum, $T_{\mathrm{SI}}(\lambda)$, operate in a linear region, and expressions for spectral gain and sensibility are also discovered. AOM has a bandwidth from $0.1 \mathrm{~Hz}$ to $20 \mathrm{kHz}$, and its dynamic range is from 0.0 to $43.5 \mathrm{~dB}$.
\end{abstract}

\section{Introduction}

Photonic crystal fibres (PCFs) are a class of optical fibres that have generated a lot of research interest in recent years [1]. The flexibility in the design of PCFs distinguishes them from conventional fibres, and various PCFs have been developed targeting different applications such as fibre-optic based sensing $[2,3]$. Polarization-maintaining photonic crystal fibres (PM-PCFs) have become commercially available nowadays; these PCFs have important features such as high birefringence and low temperature sensitivity. Up to now, the PM-PCFs implemented in loops of Sagnac interferometers (SI) have worked properly for purposes of detecting mechanical stress and temperature variations [4]. Particularly, this acoustooptic modulator offers an input port to the constant power CW beam $P_{\text {in }}\left(\lambda_{0}\right)$ and an output port, through which the modulated beam emerges, $P_{\text {out }}\left(\lambda_{0}, t\right)$, ready to get to the end of the fibre-optic line to reach the receiver stage (PD). In this work mainly, we present an all-optical fibre acoustooptic modulator based on SI with PM-PCF loop, perfectly adjusted to operate in the C band (1530-1570 nm) or in the L band (1570-1620 nm) for optical communications. Now, we will demonstrate that SI of PM-PCF loop, under specific conditions, is able to work as a linear acoustooptic laser modulator.

\section{Operation of AOM}

2.1. Theory of Sagnac Interferometer with PM-PCF Loop. Sagnac interferometer (SI) is made up of a single mode fibre (SMF), $3 \mathrm{~dB}$ coupler for $1550 \mathrm{~nm}$, and a segment of PMPCF (PM-1550-01, Thorlabs ${ }^{\circledR}$ ) which interconnects the two output ports of coupler forming the loop Sagnac of length $L$, as shown in Figure 1(a). To find the spectral response of SI, an infrared source spectrum is used with a flat intensity distribution in the vicinity of $1550 \mathrm{~nm}$. This produces an output spectrum with cosine profile, which is measured with an optical spectrum analyser (OSA). Transmittance function of SI is as follows [5-8]:

$$
T_{\mathrm{SI}}=\frac{P_{\text {out }}(\lambda)}{P_{\text {in }}(\lambda)}=\frac{1}{2}\left[1+\cos \Delta \varphi_{n v}\right],
$$




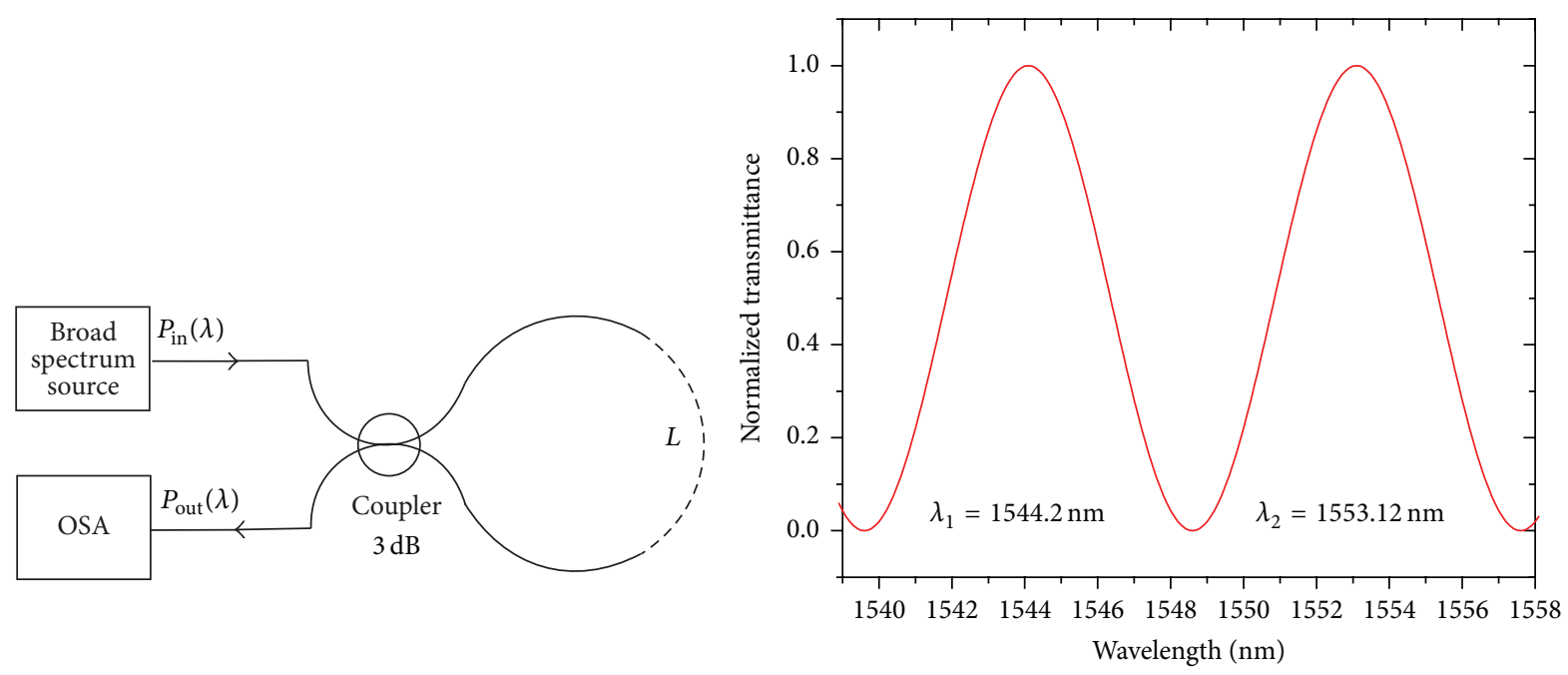

(a)

(b)

Figure 1: (a) All-fibre Sagnac interferometer with PM-PCF loop, L. (b) Typical transmittance spectrum of SI.

where $\Delta \varphi_{n v}$ is represented by

$$
\Delta \varphi_{n v}=\frac{2 \pi}{\lambda} \Delta n_{n v} L
$$

where $\Delta n_{n v}=n_{x n v}-n_{y n v}$, where $n_{x n v}$ and $n_{y n v}$ are the corresponding refractive index of each birefringent fibre axis. Subindex $n v$ means that the variable is valuated in the absence of mechanical stress and at laboratory temperature, $21^{\circ} \mathrm{C}$. Thus, it is necessary to know $\Delta n_{n v}$ of PM-PCF to make a specific characterization of SI beforehand. This is possible by using

$$
\Delta n_{n v}=\frac{\lambda^{2}}{\Delta \lambda} \frac{1}{L},
$$

where $\Delta \lambda=\lambda_{2}-\lambda_{1}$, where $\lambda_{1}$ and $\lambda_{2}$ are wavelengths corresponding to a couple of adjacent maximum (or minimum) of the transmittance spectrum $T_{\mathrm{SI}}(\lambda)$ shown in Figure 1(b). $\lambda$ is the average value between $\lambda_{1}$ and $\lambda_{2}$. Likewise, (4) is useful for calculating the beat length of the birefringence fibre:

$$
L_{B}=\frac{\lambda}{\Delta n_{n v}} .
$$

2.2. Description and Operation of the AOM System. The experimental setup to demonstrate the functionality of AOM is shown in Figure 2. One can see that the AOM system consists of a sine wave generator (Gen) to excite a speaker of 100 watts RMS with tones from $0.1 \mathrm{~Hz}$ to $25 \mathrm{kHz}$ at a uniform intensity of $10 \mathrm{~dB}$. The orthogonal distance between the speaker and the birefringent loop is $30 \mathrm{~cm}$. The AOM system has SI as an important part, which is irradiated with CW-ILD $1550 \mathrm{~nm}$ by its input port with constant power $P_{\text {in }}\left(\lambda_{0}\right)(1-5 \mathrm{mw}) . P_{\text {in }}\left(\lambda_{0}\right)$ will be modulated by the spectral shifts of $T_{\mathrm{SI}}(\lambda)$ dependent on induced vibration modes in the
PM-PCF segment of length $L$. It should be mentioned that the birefringent segment, $L$, is placed within a soundboard and is tense at the ends by using a couple of twisters, with strength of $200 \mathrm{gr}$, enough to keep it in straight line, so that only a portion $L_{v}$ is exposed to vibration emitted by the speaker, $L_{v}<L$. As a result of laser modulation, the output signal $P_{\text {out }}\left(\lambda_{0}, t\right)$ is generated, which affects the InGaAs photodetector (PD) to be converted into an electrical signal. This electrical signal enters the audio amplifier, whose output is recognized as the output of the AOM system. Finally, this signal is sent for its recovery, measurement, and analysis together with Gen signal to the same oscilloscope, as shown in Figure 2.

The PM-PCF employed in this SI had the following characteristics: pitch $\Lambda$ (spacing between holes): $4.4 \mu \mathrm{m}$, large hole diameter: $4.5 \mu \mathrm{m}$, small hole diameter: $2.2 \mu \mathrm{m}$, diameter of holey region: $40.0 \mu \mathrm{m}$, and outside diameter: $125 \mu \mathrm{m}$ (see Figure 3).

A physical response of the birefringent fibre is that its birefringence $\Delta n_{n v}$ is modified when the fibre experiences mechanical stress or variations of temperature, and then its resulting birefringence, $\Delta n_{r}$, can be described like $\Delta n_{n v} \pm$ $\Delta n_{v}$ [9]. $\Delta n_{i}$ represents the induced birefringence on fibre. Consequently, this response produces a spectral shifting of transmittance function of Sagnac interferometer. The transmittance shifting starts from its initial position towards the short wavelengths, in proportion of the excitation energy, and returns to its initial position, when the excitation energy disappears. Taking into account these conditions, a model of the phase function, $\Delta \varphi\left[i_{m}(t)\right]$, dependent on the mechanical vibration of the birefringent segment, $i_{m}(t)$, which is induced by the incident acoustic waves can be formulated.

2.3. Frequency Response of $A O M$ : $H(\omega)$. The mathematical model of the operation of the acoustooptic modulator (AOM) starts considering that the incident acoustic signals are 


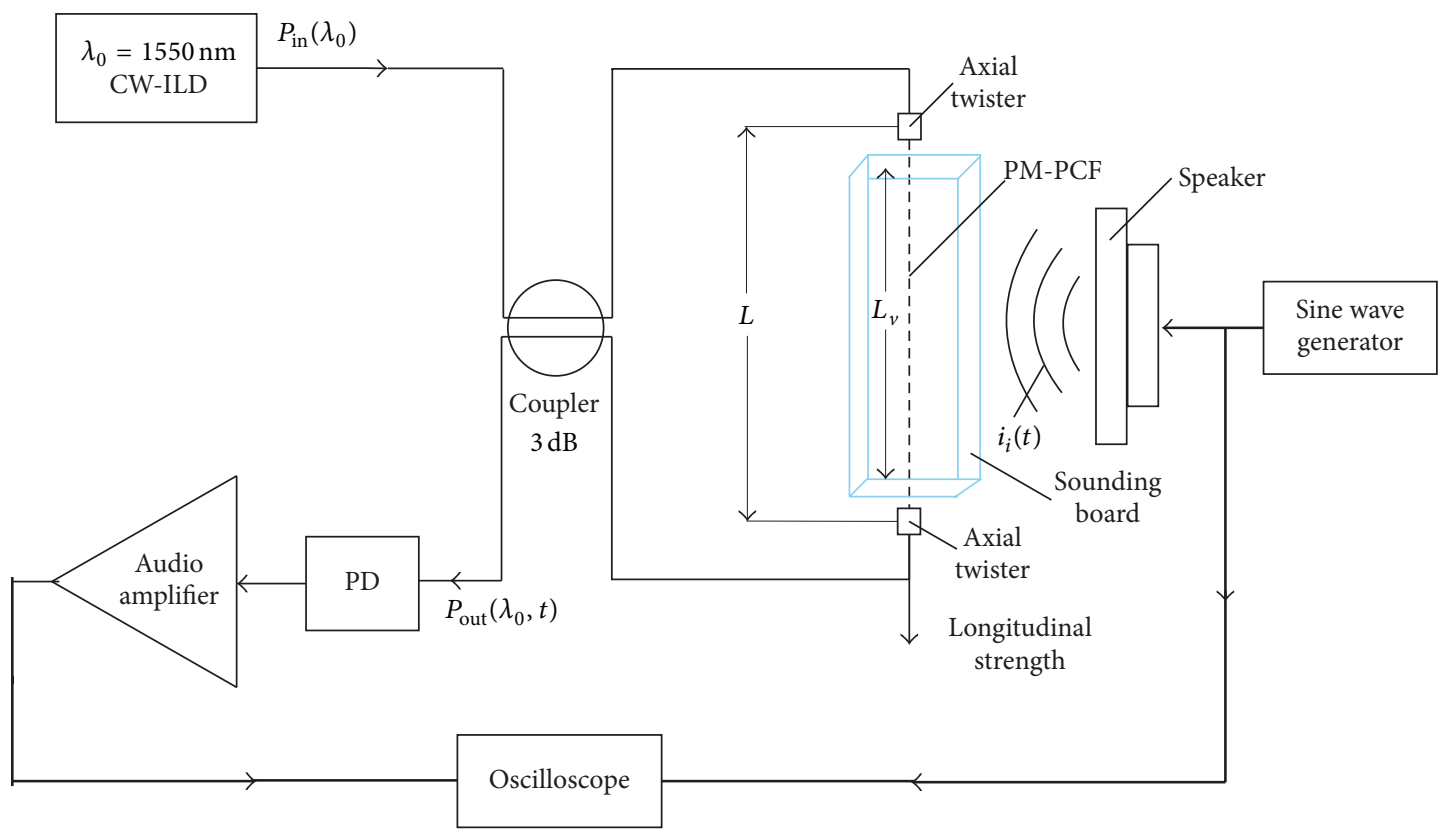

FIGURE 2: Acoustooptic modulator setup based on Sagnac interferometer with oscillating segment $L_{v}$.

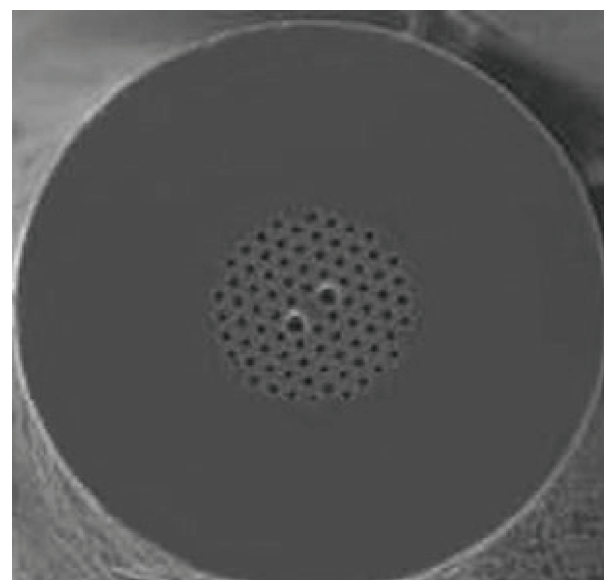

FIgURE 3: Cross-sectional SEM image of the PM-PCF.

changes of pressure, represented by $i_{i}(t)=i_{c}+i_{v}(t)$, where $i_{c}$ is the constant intensity component and $i_{v}(t)$ is the timevariant intensity component. In this application, $i_{i}(t)$ belongs to the intensity of a mechanical wave in the acoustic range. When this wave hits the Sagnac loop, $i_{i}(t)$ becomes as shown in

$$
i_{m}(t)=\beta i_{i}(t),
$$

where $\beta$ is the fraction of energy $i_{i}(t)$, which is converted to $i_{m}(t) . i_{m}(t)$ represents energy intensity modulation induced in the Sagnac interferometer loop. By the Fourier transform, $i_{m}(t)$ has a spectral representation $I_{m}(\omega)$, as follows:

$$
i_{m}(t) \stackrel{\mathbb{F}}{\leftrightarrow} I_{m}(\omega) .
$$

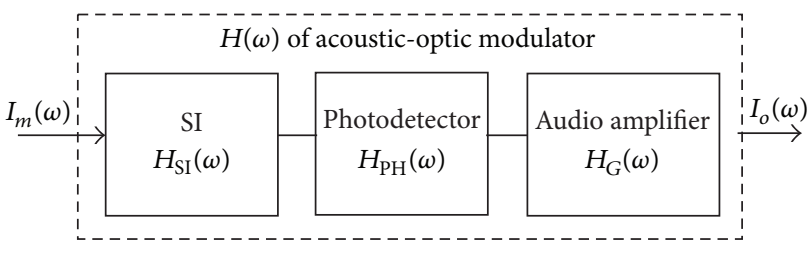

FIGURE 4: The resulting transfer function acoustooptic modulator, $H(\omega)$, is represented by the multiplication of the transfer functions of the Sagnac interferometer, the photodiode, and the audio amplifier.

$I_{m}(\omega)$ is a continuous spectrum because any induced $\omega$ produce expansions and contractions, to a greater or lesser extent, to the structure of the birefringent fibre loop, so $\Delta n$ values are modulated. Characteristics of $I_{m}(\omega)$ influence the modulator bandwidth. $I_{m}(\omega)$ is the input continuous spectrum of AOM, which produces a continuous output optical spectrum, $I_{o}(\omega)$. The relationship between them is shown in

$$
I_{o}(\omega)=H(\omega) \cdot I_{m}(\omega),
$$

where $H(\omega)$ is the resulting transference function or filter response of acoustooptic modulator. Before $I_{m}(\omega)$ reaches the output, the system has to go through the transfer functions of $H_{\mathrm{SI}}(\omega)$ and $H_{\mathrm{PH}}(\omega)$ and then through the audio amplifier $H_{G}(\omega)$, as shown in Figure 4.

The transference function of linear modulator, $H(\omega)$, is defined in

$$
H(\omega)=H_{\mathrm{SI}}(\omega) \cdot H_{\mathrm{PH}}(\omega) \cdot H_{G}(\omega) .
$$


The term $H_{\mathrm{SI}}(\omega)$ is defined according to

$$
H_{\mathrm{SI}}(\omega)=a_{0} \exp \left(-\alpha_{1} \omega^{2}\right) \sin ^{2}\left[\frac{2 \pi}{\Delta \omega} \omega\right] \cdot \exp \left(-j \omega t_{0}\right),
$$

which represents the frequency response of SI. $a_{0}$ is the maximum value reached at the IS output. $\alpha_{1}$ allows us to adjust the extinction ratio of resonance maximums of $H_{\mathrm{SI}}(\omega)$. The quadratic sinusoidal behaviour appears due to the permitted oscillations of birefringent fibre segment tensed by the extremes, $L_{v}$. $\Delta \omega$ represents resonance mode separation. The term $-j \omega t_{0}$ represents the phase shift between the output and the input signals, and $t_{0}$ represents the delay between the output and the input signals. The term $H_{\mathrm{PH}}(\omega)$ is defined according to

$$
H_{\mathrm{PH}}(\omega)=\frac{K}{j a_{1} \omega+1},
$$

which represents the spectral response of the photodetector, which corresponds to a first-order filter. $K$ represents the gain of the filter, and $a_{1}$ regulates the degree of phase shift between input and output signals, $\theta_{\mathrm{PH}}$. Moreover, $a_{1}$ allows us calculate the cut-off frequency $\omega_{c}=1 / a_{1}$; the photodiode rise time is $2 \mu \mathrm{s}$; then $\omega_{c}=1 \mathrm{MHz}$-rad; we can consider that $H_{\mathrm{PH}}(\omega)$ has a predominantly planar response, so that $H_{\mathrm{PH}}(\omega)$ does not present notable dependence on $\omega$, thus contributing like a constant to $H_{\mathrm{PH}}(\omega)$, only. $H_{G}(\omega)$ represents a planar frequency response of audio amplifier gain in the acoustical spectral region, as is shown in

$$
H_{G}(\omega)=a_{2} \exp \left(-j \omega t_{1}\right)
$$

where $a_{2}$ is the gain in the audio range. The term $-j \omega t_{1}$ represents the phase shift between the output and the input signals, and $t_{1}$ represents the delay between the output and the input signals. The maximum resonant modes can be calculated up to $\pm m$ th harmonic, so that $\omega_{1}=2 \pi f_{1}=\Delta \omega$, which represents the first vibration mode of birefringent fibre tensed by the ends. $f_{1}$ is compatible with the mode of PMPCF tensed by the extremes, through

$$
f_{m}=\frac{m}{2 L_{v}} v
$$

where $v=\left(T / \rho_{L}\right)^{1 / 2}$ is wave propagation speed in the length segment $L_{v}$ of PM-PCF with tension $T$ and linear mass density $\rho_{L}$. Finally, the transference function of the complete system $H(\omega)$, which contains the information about the magnitude and phase response of AOM, is shown in

$$
\begin{aligned}
H(\omega)= & a_{0} \exp \left(-\alpha_{1} \omega^{2}\right) \sin ^{2}\left[\frac{2 \pi}{\Delta \omega} \omega\right] \\
& \cdot \exp \left[-j\left(\omega\left(t_{0}+t_{1}\right)-\tan ^{-1}\left(a_{1} \omega\right)\right)\right] .
\end{aligned}
$$

Consequently, $H(\omega)$ expresses both the proportion in which the intensity of the vibration modes is induced in the loop and how these contribute to the modulation of the birefringence value $\Delta n$, and it also shows the filter response of AOM.
2.4. Acoustooptic Modulator Transmittance Model. To obtain the function of transmittance in dependence on the induced transversal vibration modes in the segment, $L_{v}$, of the birefringence fibre of length $L$, we must consider the phase changes, $\Delta \varphi$, produced through the loop segments: $L-L_{v}$ and $L_{v}$. On the basis of our experimental results, the resulting change rapidity of refraction index of both axes $n_{r x}$ and $n_{r y}$ in the birefringent fibre with regard to the induced vibration, $i_{m}(t)$, is predominantly linear, as is expressed in

$$
\begin{aligned}
& n_{r x}=n_{x n v}+\frac{\partial n_{x v}}{\partial i_{m}} \cdot i_{m}, \\
& n_{r y}=n_{y n v}+\frac{\partial n_{y v}}{\partial i_{m}} \cdot i_{m},
\end{aligned}
$$

where $n_{x n v}$ and $n_{y n v}$ are the refraction indexes in $x$ and $y$ in the absence of vibration and $n_{x v}$ and $n_{y v}$ are the refraction indexes induced by $i_{m}(t)$ to each of the axes. It is emphasized that this analysis is realized to constant temperature of laboratory. Thus, given that $\Delta n_{r}=n_{r x}-n_{r y}$ and $\Delta n_{v}=$ $n_{x v}-n_{y v}$, the expression of $\Delta n_{r}$ is shown in

$$
\Delta n_{r}=\Delta n_{n v}+\frac{\partial \Delta n_{v}}{\partial i_{m}} \cdot i_{m}
$$

where

$$
\frac{\partial \Delta n_{v}}{\partial i_{m}}=\delta_{\mathrm{disp}}
$$

where $\delta_{\text {disp }}$ is the induced birefringence dispersion, which is proportional to the acoustooptic modulator gain, and represents the birefringence changes rapidity of PM-PCF with regard to contained oscillation modes in $i_{m}(t)$. From (2), the total phase difference reached across $L$ is obtained by adding algebraically the phase differences $\Delta \varphi_{n v}$ and $\Delta \varphi_{i}$ in their respective segments $L-L_{v}$ and $L_{v}$, as shown in

$$
\begin{aligned}
\Delta \varphi\left[i_{m}(t)\right] & =\Delta \varphi_{n v}+\Delta \varphi_{i} \\
& =\frac{2 \pi}{\lambda} \Delta n_{n v} \cdot\left(L-L_{v}\right)+\frac{2 \pi}{\lambda} \Delta n_{i} \cdot i_{m}(t) \cdot L_{v}
\end{aligned}
$$

which, by replacing (15) and (16) in (17) and regrouping, becomes

$$
\Delta \varphi\left[i_{m}(t)\right]=\frac{2 \pi}{\lambda}\left(\Delta n_{n v} L+\delta_{\mathrm{disp}} i_{m}(t) L_{v}\right)
$$

phase changes due to $\Delta \varphi\left[i_{m}(t)\right]$ are hardly amplified in the measure where $L_{v}$ increases. Equation (18) will serve to adjust the experimental response of modulator towards operation point, gain, and appropriated sensibility. Thus, $T\left[i_{m}(t)\right]$ of AOM for $\lambda_{0}$ is described through

$$
\begin{aligned}
T\left[i_{m}(t)\right]_{\mathrm{SI}}= & \frac{1}{2} \\
& +\frac{1}{2} \cos \left(\frac{2 \pi}{\lambda_{0}}\left[\Delta n_{n v} L+\delta_{\mathrm{disp}} i_{m}(t) L_{v}\right]\right)
\end{aligned}
$$




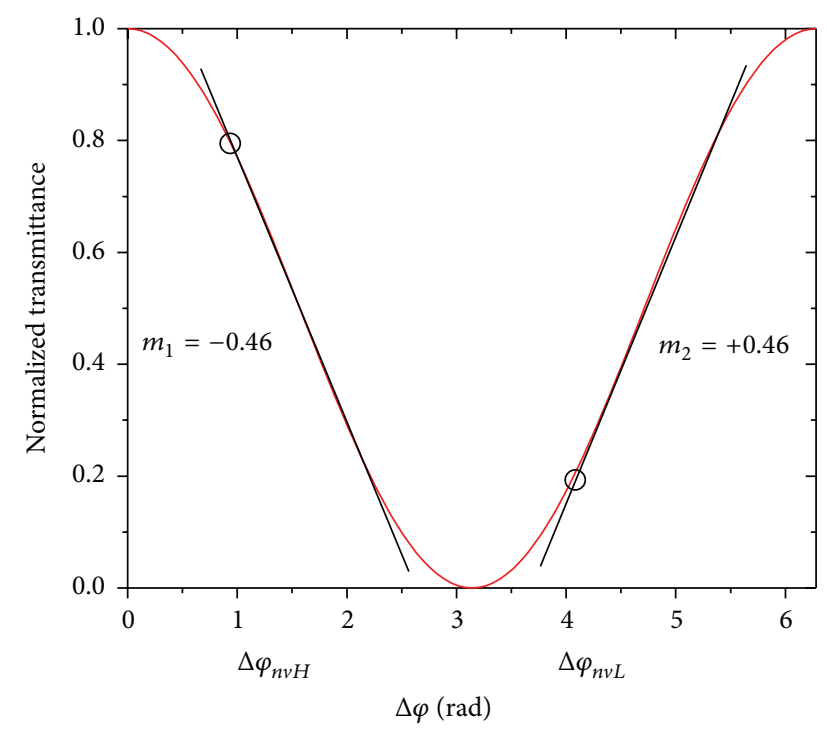

FIGURE 5: Two operation points: $\left(\Delta \varphi_{n v L}, 0.2\right)$ or $\left(\Delta \varphi_{n v H}, 0.8\right)$ with their tangent lines whose slopes are $m_{1,2}= \pm 0.46$, respectively.

and spectral shifting rapidity of $T\left[i_{m}(t)\right]$ due to $i_{m}(t)$ is calculated through

$$
\frac{\partial \lambda\left(i_{m}\right)}{\partial i_{m}}=\frac{\lambda_{0} \delta_{\text {disp }}}{\Delta n_{n v}} \cdot \frac{L_{v}}{L} .
$$

The modulation capacity of the interferometer depends on the frequency of the induced vibration modes, $i_{m}(t)$. This capacity depends proportionally on the birefringence dispersion, $\delta_{\text {disp. }}$. Shifting rapidity can be amplified, when the measure of relationship $\left(L_{v} / L\right)$ is adjusted, propitiating an increase in the sensibility of acoustooptic modulator for each vibration frequency of the tensed PM-PCF by the ends.

2.5. Operation Point Adjustment of AOM. The variable $\Delta \varphi_{0}$ represents the phase value from which the phase values of the Sagnac interferometer transmittance will be increased. The incident of the wave acoustics produces a phase increase $\Delta \varphi_{v}\left(i_{m}\right)$, which implies a spectral shifting for $T_{\mathrm{SI}}\left[i_{m}(t)\right]$ towards the short wavelengths. In phase terms, there exist two main zones to make linear modulation in the transmittance curve, as shown in Figure 5. Two possible values exist for $\Delta \varphi_{n v}$ as operation point: $\Delta \varphi_{n v H}$ or $\Delta \varphi_{n v L}$. So, the value of $\Delta \varphi_{n v}$ must be adjusted to one of these values, at $21^{\circ} \mathrm{C}$, for $\lambda_{0}=1550 \mathrm{~nm}$, and in the absence of induced vibration, $i_{m}(t)=0 . \Delta \varphi_{n v}$ must reach one of these phase values, like an operation point. Numerical values of these phases are $\Delta \varphi_{n v H}=(9 / 25) \pi+k \cdot 2 \pi$ and $\Delta \varphi_{n v L}=(13 / 10) \pi+k \cdot 2 \pi$, where $k$ is an entire number. These values should be located strictly in the wavelength of operation, $\lambda_{0}$, just at the beginning of one of the linear modulation regions (see Figure 5). Thus, it is said that $T_{\mathrm{SI}}\left[i_{m}(t)\right]$ is in its operation point, as is shown in

$$
T_{\mathrm{SI}}\left[i_{m}(t)\right]=\frac{1}{2}+\frac{1}{2} \cos \left(\Delta \varphi_{n v}+\Delta \varphi_{v}\left(i_{m}(t)\right)\right) \text {. }
$$

It is important to make a good choice of $L$ value, in order to produce an appropriated operation point in $T_{\mathrm{SI}}\left[i_{m}(t)\right]$.
2.6. Laser Modulation Equation. The modulator is mainly constituted by Sagnac interferometer with high birefringence PM-PCF, in which transmittance function, before acoustic stimulus, responds with spectral shifting forward short wavelengths. Thus, the input and output optical power have the following relationship:

$$
P_{\text {out }}\left(\lambda_{0}, t\right)=T_{\mathrm{SI}}\left(\lambda_{0}, i_{m}(t)\right) \cdot P_{\text {in }}\left(\lambda_{0}\right)
$$

where modulating function is the transmittance $T_{\mathrm{SI}}\left(\lambda_{0}\right.$, $\left.i_{m}(t)\right)$. Thus, $T_{\mathrm{SI}}\left(\Delta \varphi_{n v}\right)$ must have a point operation with the next specifications: $\Delta \varphi_{n v}=\Delta \varphi_{n v H}$ or $\Delta \varphi_{n v}=\Delta \varphi_{n v L}$ at $21^{\circ} \mathrm{C}$, for $\lambda_{0}=1550 \mathrm{~nm}$ laser with constant power, $P_{\text {in }}\left(\lambda_{0}\right)$, and in the absence of induced vibration, $i_{m}(t)$. Consequently, $T_{\mathrm{SI}}\left(\Delta \varphi_{n v}\right)$ has to be equal to 0.8 or 0.2 , respectively. Operation points have coordinate points $\left(\Delta \varphi_{n v L}, 0.2\right)$ or $\left(\Delta \varphi_{n v H}, 0.8\right)$ and are represented by circles, as shown in Figure 5 . The straight lines with $m_{1}$ and $m_{2}$ slopes represent the linear modulation regions. Thus, two kinds of modulation exist.

2.6.1. Negative Modulation. For the behaviour of $T_{\mathrm{SI}}\left(\lambda_{0}\right.$, $\left.\Delta \varphi_{n v H}, i_{m}(t)\right)$ in the region $\lambda_{0}<\lambda$, its cosine profile can be approximated with tangent linear equation at the operation point $P\left(\Delta \varphi_{n v H}, 0.8\right)$, where positive slope, $m$, is expressed in

$$
\begin{aligned}
T_{\mathrm{SI}}\left[i_{m}(t)\right] & =\frac{1}{2}+\frac{1}{2} \cos \left(\Delta \varphi_{n v}+\Delta \varphi_{v}\left(i_{m}\right)\right) \\
& \approx \frac{\partial T_{\mathrm{SI}}}{\partial \Delta \varphi_{v}} \cdot\left(\Delta \varphi_{v}-\Delta \varphi_{n v H}\right)+\frac{8}{10},
\end{aligned}
$$

where $m_{1}=\partial T_{\mathrm{SI}} / \partial \Delta \varphi_{v}=-0.46$ is the negative slope in the middle region between the crest and valley of function cosine.

2.6.2. Positive Modulation. For the behaviour of $T_{\mathrm{SI}}\left(\lambda_{0}\right.$, $\left.\Delta \varphi_{n v L}, i_{m}(t)\right)$ in the region $\lambda_{0}<\lambda$, its cosine profile can be approximated with tangent linear equation at the operation point $P\left(\Delta \varphi_{n v L}, 0.2\right)$, where positive slope, $m$, is expressed in

$$
\begin{aligned}
T_{\mathrm{SI}}\left[i_{m}(t)\right] & =\frac{1}{2}+\frac{1}{2} \cos \left(\Delta \varphi_{n v}+\Delta \varphi_{v}\left(i_{m}\right)\right) \\
& \approx \frac{\partial T_{\mathrm{SI}}}{\partial \Delta \phi_{v}} \cdot\left(\Delta \varphi_{v}-\Delta \varphi_{n v L}\right)+\frac{2}{10},
\end{aligned}
$$

where $m_{2}=\partial T_{\mathrm{SI}} / \partial \Delta \varphi_{v}=+0.46$ is the positive slope in the middle region between the crest and valley of function cosine. In both cases, in linear region, we have the following:

$$
0<\Delta \varphi_{v}-\Delta \varphi_{n v x} \leq \frac{4}{3} \mathrm{rad}
$$

where $x$ can be $H$ or $L$. Thus, laser modulation in nonlinear regions is avoided. Phase relationship between $i_{m}(t)$ and $P_{\text {out }}\left(\lambda_{0}, t\right)$ is $\pi \mathrm{rad}$ for negative modulation and $0.0 \mathrm{rad}$ for positive modulation.

From the spectral point of view, linear modulation occurs because $T_{\mathrm{SI}}(\Delta \varphi)$ undergoes a proportional shift towards short wavelengths, to the magnitude of the induced acoustic signal, $i_{m}(t)$, returning to its initial position, as this disappears. This modulation process is represented in the diagrams shown 


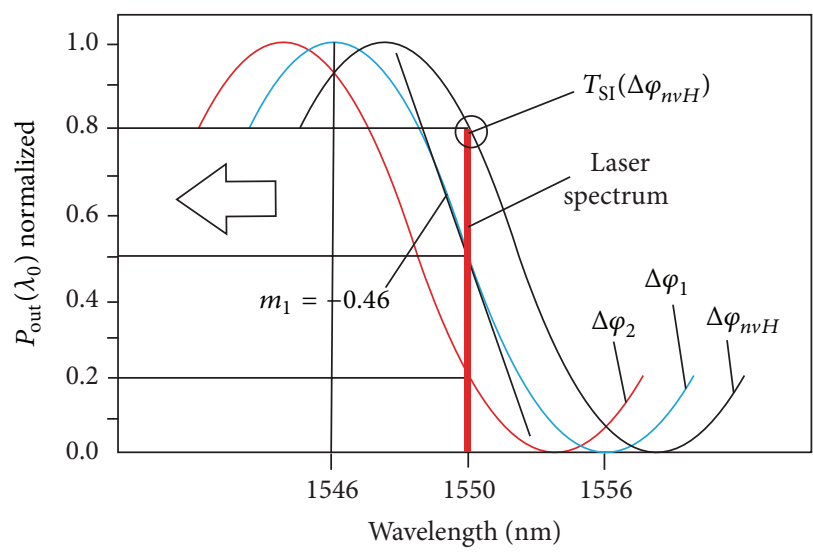

(a)

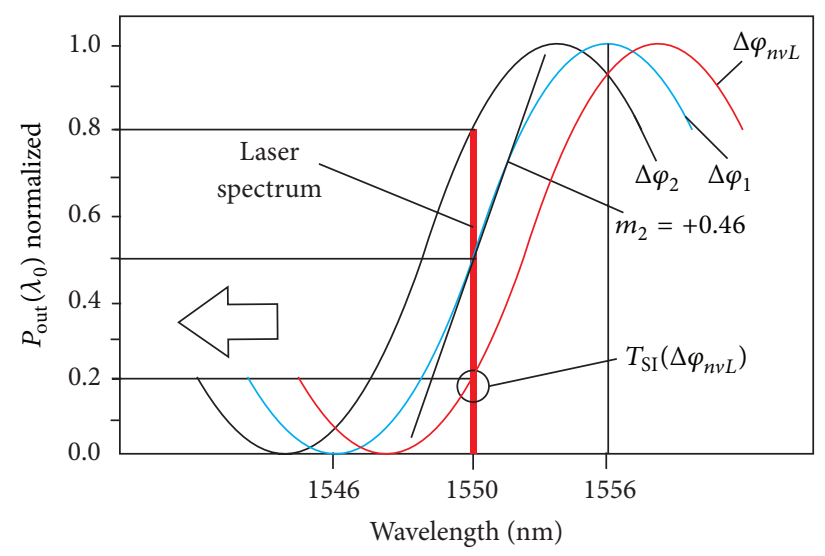

(b)

FIGURE 6: (a) Operation point of negative modulation; (b) operation point of positive modulation.

in Figures 6(a) and 6(b). Note that operation points are $T_{\mathrm{SI}}\left(\Delta \varphi_{n v H}\right)=0.8$ with $\Delta \varphi_{n v H}<\Delta \varphi_{1}<\Delta \varphi_{2}$ for case (a) and $T_{\mathrm{SI}}\left(\Delta \varphi_{n v L}\right)=0.2$ with $\Delta \varphi_{n v L}<\Delta \varphi_{1}<\Delta \varphi_{2}$ for case (b). The linear modulation process happens when the linear region of the $T_{\mathrm{SI}}(\Delta \varphi)$ cosine profile regulates the intensity of the CWILD laser beam with its shifting and this effect is reflected in the reproduction of the form of $i_{m}(t)$ in $P_{\text {out }}\left(\lambda_{0}, t\right)$ values.

2.7. Sensibility of AOM. The laser linear modulator, as a linear time-invariant system, is admitted as input signal to resonant signal, $i_{m}(t)$, which is induced in the segment of PM-PCF, and as an output signal to $P_{\text {out }}\left(\lambda_{0}, t\right)$, as a result of spectral displacement of $T_{\mathrm{SI}}\left(\lambda_{0}, i_{m}\right)$, which modulates $P_{\text {in }}$. This modulation is proportional to shifting rapidity of $T_{\mathrm{SI}}\left(\lambda_{0}, i_{m}\right)$, due to the changes of $\Delta \varphi_{v}\left(i_{m}\right)$. Thus, sensibility of AOM can be defined, as expressed in

$$
S=\frac{\partial P_{\text {out }}}{\partial i_{m}}
$$

Replacing (22) in (26) yields

$$
S=P_{\text {in }} \cdot \frac{\partial T_{\mathrm{SI}}\left(\lambda_{0}\right)}{\partial \Delta \varphi_{v}} \cdot \frac{\partial \Delta \varphi_{v}}{\partial i_{m}},
$$

where $P_{\text {in }}\left(\lambda_{0}\right)$ appears as a constant term which is modulated by Sagnac interferometer transmittance. The expression $\partial T_{\mathrm{SI}}\left(\lambda_{0}\right) / \partial \Delta \varphi_{v}$ characterizes the change rapidity of the transmittance due to the induced phase changes by acoustic signal. Because of the trigonometric properties of $T_{\mathrm{SI}}(\lambda)$ function, its linear region of modulation has an intrinsic corresponding slope $m$, which is described by

$$
m_{1,2}=\frac{\partial T_{\mathrm{SI}}\left(\lambda_{0}\right)}{\partial \Delta \varphi_{v}}=\mp 0.46,
$$

where the sign of slope depends on the side of the crest which is employed to realize the modulation. The expression $\partial \Delta \varphi_{v} / \partial i_{m}$ represents the sensibility to the induced phase change by induced signal $i_{m}$, in the birefringent fibre:

$$
\frac{\partial \Delta \varphi_{v}}{\partial i_{m}}=\frac{2 \pi}{\lambda_{0}} \delta_{\text {disp }} L_{v}
$$

TABLE 1: Optical parameters of PM-PCF.

\begin{tabular}{lcc}
\hline & Measured parameters experimentally \\
$\Delta n_{n v}$ & $L_{B}, \mathrm{~mm}$ & $\delta \lambda / \delta i_{m}, \mathrm{~nm} / \mathrm{dB}$ \\
\hline $7.6 \times 10^{-4}$ & 2.0 & 1 \\
\hline
\end{tabular}

Let us observe that both $\delta_{\text {disp }}$ and $L_{v}$ perform a relevant role in the adjustment of sensibility and denote that sensibility to the phase change also depends on intensity and vibration frequencies, $I_{m}(\omega)$. This final result provokes the notion that the sensibility of AOM is high in accordance with the acoustic noise and with the external vibration of the workbench.

\section{Experimental Results and Discussion}

3.1. Required Characteristics for $T_{S I}(\lambda) . \Delta n_{n v}$ and $L_{B}$ parameters are determined by using (3) and (4) [9]. Consider that we intend to design and build a SI, with loop PM-PCF, whose $T_{\mathrm{SI}}(\lambda)$ without mechanical excitation and at $21^{\circ} \mathrm{C}$ offers a spectral distribution, such that $T_{\mathrm{SI}}\left(\lambda_{0}\right)=0.2$ to make positive modulation. Moreover, consider that the shifting ratio of $T_{\mathrm{SI}}(\lambda)$ towards shorter wavelengths, with acoustical excitation, is $1 \mathrm{~nm} / \mathrm{dB}$ for a component of $1 \mathrm{kHz}$, when $\left(L_{v} / L\right)=1$ typically. Consequently, $T_{\mathrm{SI}}(\lambda)$ should have $\Delta \lambda=$ $10 \mathrm{~nm}$ to have at least $4 \mathrm{~nm}$ of linear modulation region. In consideration of the characterization of the PM-PCF, Table 1 shows the experimentally measured optical parameters and Table 2 shows the spectral characteristics of the built SI.

We can show as a result of the spectral measurements that the overlapping spectra of SI transmittance and of emission of ILD $\left(\lambda_{0}=1550 \mathrm{~nm}\right)$ are shown in Figure 7, observing that the location of the spectrum of ILD is right where $T_{\mathrm{SI}}\left(\lambda_{0}\right)=$ 0.2 , guaranteeing the right conditions to perform positive acoustooptic modulation.

3.2. Modulator Response in Time and Frequency Domain. Time response of acoustooptic modulator was determined by using the setup procedure shown in Figure 2. An electrical sine wave, $i_{i}(t)$, is applied in the speaker by using wave 
TABLE 2: Physical and spectral characteristics of SI.

\begin{tabular}{cccccccc}
\hline \multicolumn{8}{c}{ Characteristic values obtained } \\
$L, \mathrm{~m}$ & $L_{v}, \mathrm{~m}$ & $\lambda_{1}, \mathrm{~nm}$ & $\lambda_{2}, \mathrm{~nm}$ & $\Delta \lambda, \mathrm{nm}$ & $\begin{array}{c}T_{\mathrm{SI}} \\
\left(\lambda_{0}\right)\end{array}$ & $\begin{array}{c}\delta \lambda / \delta i_{m}, \\
\mathrm{~nm} / \mathrm{dB}\end{array}$ & $\begin{array}{c}\Delta \varphi_{n v L}, \\
\mathrm{rad}\end{array}$ \\
\hline 0.34 & 0.19 & 1544.20 & 1553.12 & 8.9 & 0.2 & 1 & 4.0 \\
\hline
\end{tabular}

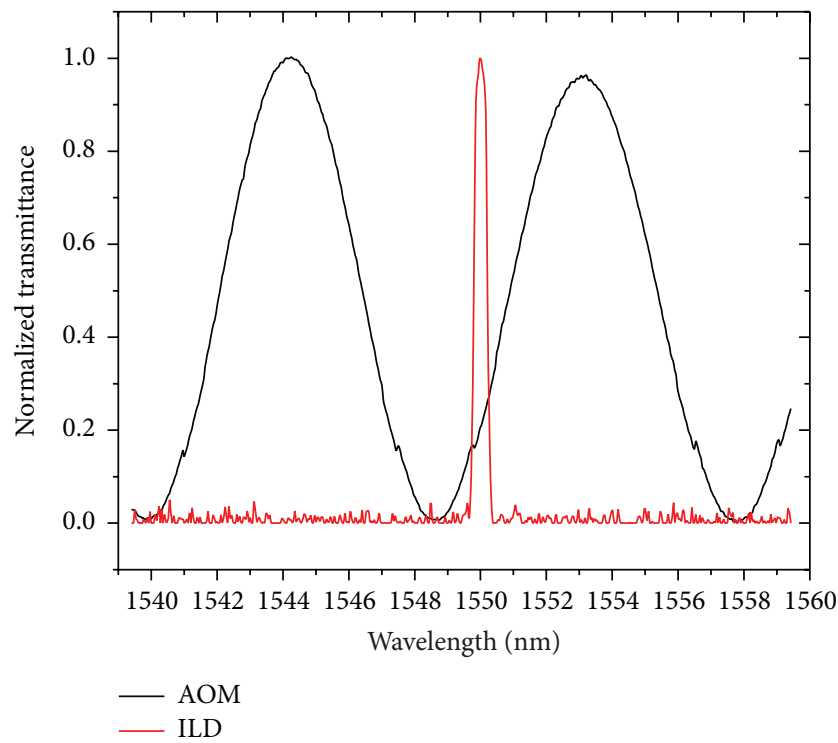

FIGURE 7: Overlapping of the normalized transmittance spectrum with the laser beam spectrum at $1550 \mathrm{~nm}$, just on the operation point $\Delta \varphi_{n v L}$.

generator (Gen), at a frequency, $f . i_{i}(t)$ at $10 \mathrm{~dB}$ is induced in the segment, $L_{v}$, like $I_{m}(t)$. According to $(22), P_{\text {out }}\left(\lambda_{0}, t\right)$ is proportionally modulated and detected by $\mathrm{PD}$. Photodetector output is amplified and represents the AOM output. Gen and AOM signals are measured and analysed in amplitude, phase, and frequency by using the oscilloscope. Some significant examples, at different frequencies, are shown in Figures 8(a), $8(\mathrm{~b}), 8(\mathrm{c})$, and $8(\mathrm{~d})$. The small phase shifting is presented due to the phase shifting provided both by photodetector and by audio amplifier. Analysis on frequency is made by using (7) and (13), determining theoretically and experimentally the Bode diagram.

Frequency response of acoustooptic modulator was determined by using the same setup shown in Figure 2. Magnitude and phase characteristics of $H_{\mathrm{SI}}(\omega)$ are hardly influenced by magnitude and phase characteristics of permitted vibration modes in the tense fibre by the ends. For this reason, gain spectrum of acoustooptic modulator shows maximum and minimum resonant peaks in audible region. Superposition of theoretical and experimental frequency response evaluated in the range from $0.1 \mathrm{~Hz}$ to $20 \mathrm{kHz}$ is shown in Figure 9. Theoretical spectral gain is reached considering a photodetector as a first-order filter, with a cut-off frequency of $175 \mathrm{kHz}$ and planar audio amplifier bandwidth of $20 \mathrm{kHz}$. We are considering that extinction ratios of resonant modes intensity are lower than the filter response of photodetector and amplifier. Separation modes were calculated for $\Delta f=7.0 \mathrm{kHz}$. Experimental gain spectrum has a lobular
TABLE 3: AOM elements.

\begin{tabular}{lc}
\hline Elements & Specifications \\
\hline ILD InGaAs & $1550 \pm 0.5 \mathrm{~nm}$, fibre pigtail, stabilized \\
Photodetector & InGaAs low power, BW $=175 \mathrm{kHz}$ \\
Amplifier & $1<$ gain $<150, \mathrm{BW}=1 \mathrm{MHz}$ \\
Modulation frequency & $0.1 \mathrm{~Hz}$ up to $21 \mathrm{kHz}$ \\
Sensibility & $2.39 \mathrm{~mW} / \mathrm{dB}$ to $1 \mathrm{kHz}$ \\
Minimum power & $1 \mathrm{~mW}$ \\
Maximum power & $5 \mathrm{~mW}$ \\
Insertion loss & $<0.4 \mathrm{~dB}$ \\
Dynamic range & $0-43.5 \mathrm{~dB}$ \\
Temperature & $21^{\circ} \pm 19^{\circ} \mathrm{C}$ \\
\hline
\end{tabular}

distribution profile, whose separation between maximums is approximately $7.0 \mathrm{kHz}$. The values of the maximums depend on the extinction ratio of the intensities of the vibration modes permitted in the segment PM-PCF, $L_{v}$. The values of the minimums depend on the sensitivity of the AOM to vibrate at frequencies not permitted.

Superposition of theoretical and experimental phase response is shown in Figure 10. It can be seen that theoretically a linear and proportional shift with respect to $\omega$ is expected, whereas the experimental result shows a shift asymptotically towards 90 degrees. The fluctuations are characteristic of the interferometer instabilities due to external vibrations outside the experiment: the ventilation system of equipment and vibration of ballast, which is very weak but existent. The differences between the theoretical and experimental response are as follows: (1) theoretical response does not include the dissipative forces of the segment $L_{v}$ (force of restitution). (2) $H_{\mathrm{SI}}$ damping coefficient depends on the operation frequency. (3) Bandwidth of every lobe is slightly different due to the fact that the losses of the vibration depend on the operation frequency value. These loss values cannot be known a priori, due to the fact that this one depends on the viscosity forces not only of the surrounding media to $L_{v}$ (Stokes law) but especially also of the friction forces at the inside of the fibre segment, $L_{v}$, for which analytical expressions do not exist. Nevertheless, this demonstrates that the optical fibre acoustooptic modulator is capable of reproducing with enough intensity the frequencies that are contained in these lobes of the gain spectrum. Let us appreciate also that the intensity of these maximums declines in the measure where the frequency increases; thus, the filter response of the modulator is similar to a low pass filter.

In spite of the fact that the optical fibre interferometers are more stable than the classic ones, the measurements of the gain spectrum were made in the absence of any other source of acoustic noise and/or vibration of the workbench. Finally, specifications of elements used for the integration of AOM to operate in the audible region, at $21^{\circ} \mathrm{C}$, are specified in Table 3.

\section{Conclusions}

In this research, we have demonstrated the feasibility of Sagnac interferometer composed of PM-PCF, which can 


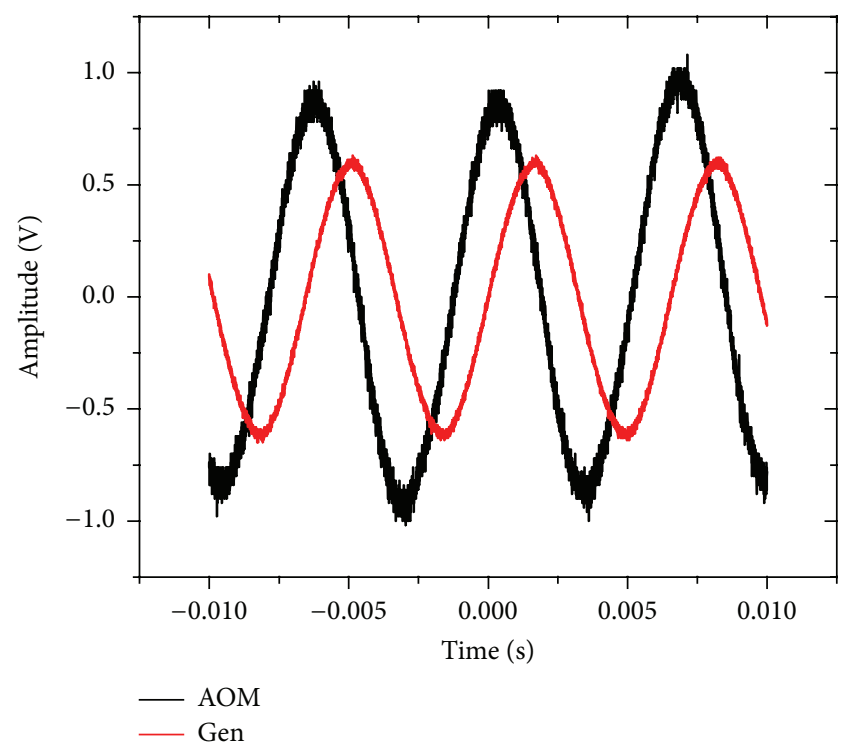

(a) $f_{m}=143 \mathrm{~Hz}$

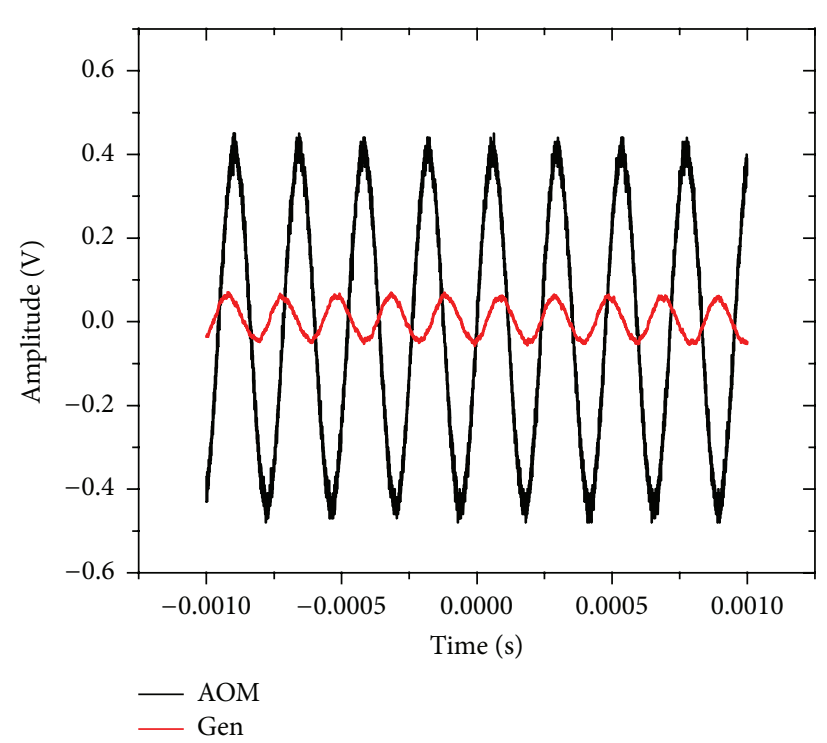

(c) $f_{m}=4.16 \mathrm{kHz}$

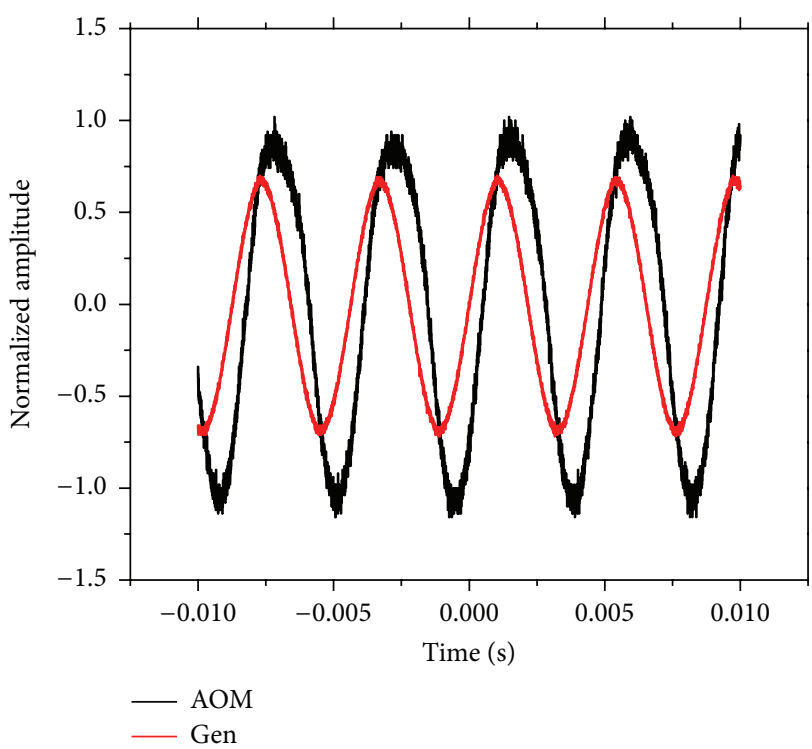

(b) $f_{m}=200 \mathrm{~Hz}$

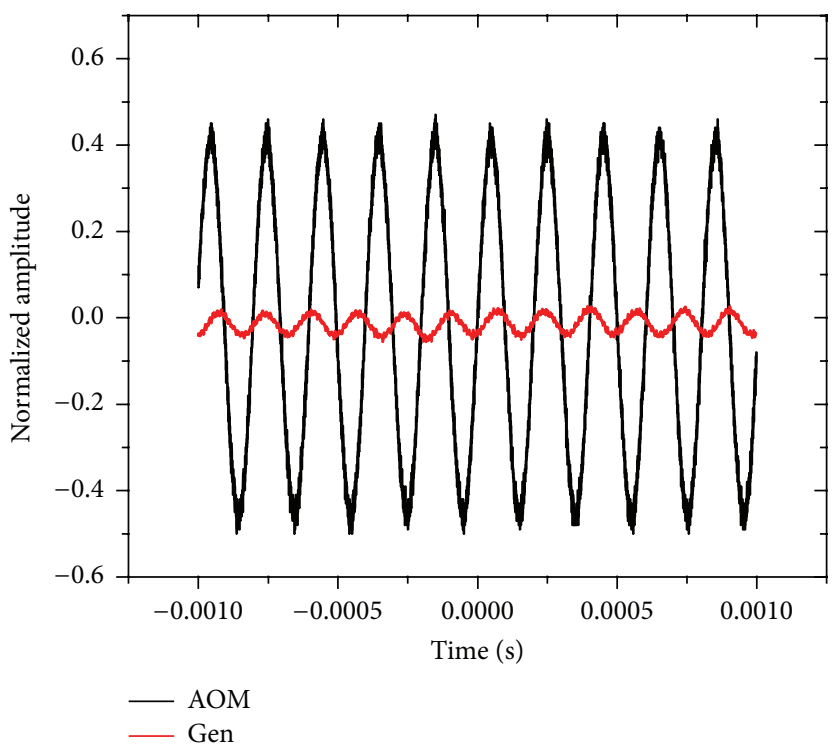

(d) $f_{m}=4.9 \mathrm{kHz}$

FIgURE 8: Input (Gen) and output (AOM) signals overlapped in oscilloscope at different frequencies.

work as a linear acoustooptic laser modulator for frequencies from $0.1 \mathrm{~Hz}$ to $20 \mathrm{kHz}$, to $1550 \mathrm{~nm}$. It has been demonstrated that the realization of the modulation, at low frequencies, of the birefringence of the PM-PCF is achievable as well. The principle of work of the AOM is that the induced shiftings in $T_{\mathrm{SI}}$, by the incident mechanical waves on the birefringent fibre, tensed by the ends, modulate the laser beam intensity. We have demonstrated the ability to linearly modulate the intensity of a CW laser beam by using the AOM. As a resonant system, it results in a gain spectrum distributed in adjacent lobes, which correspond to a response of low pass filter. We have shown that $\mathrm{AOM}$ can make positive or negative modulation. Phase relationship between acoustical signal (Gen) and optical signal (output OAM) is $\pi$ rad for negative modulation and $0.0 \mathrm{rad}$ for positive modulation. Modulator presents a wide margin of immunity to the changes of environmental temperature due to thermal characteristic of the PM-PCF loop. The dynamic range of the linear modulator is in the range of 0.0 to $43.5 \mathrm{~dB}$, enough to not reach the nonlinear region of AOM. The sensibility of the modulator depends on the frequency. Linear acoustooptic modulator can work also as a fibre microphone; we called it photonic phone. 


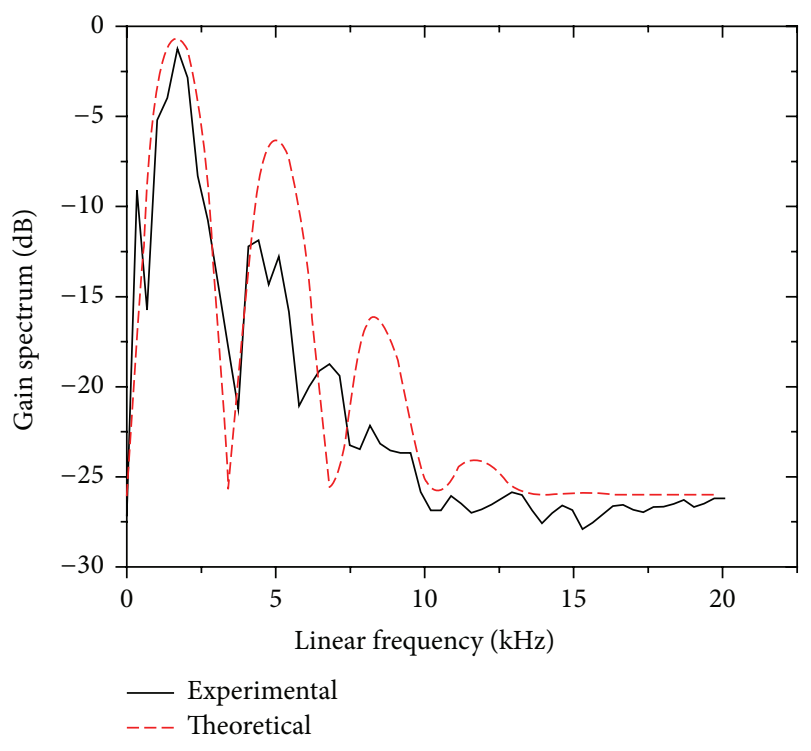

FIGURE 9: Gain spectrums of AOM.

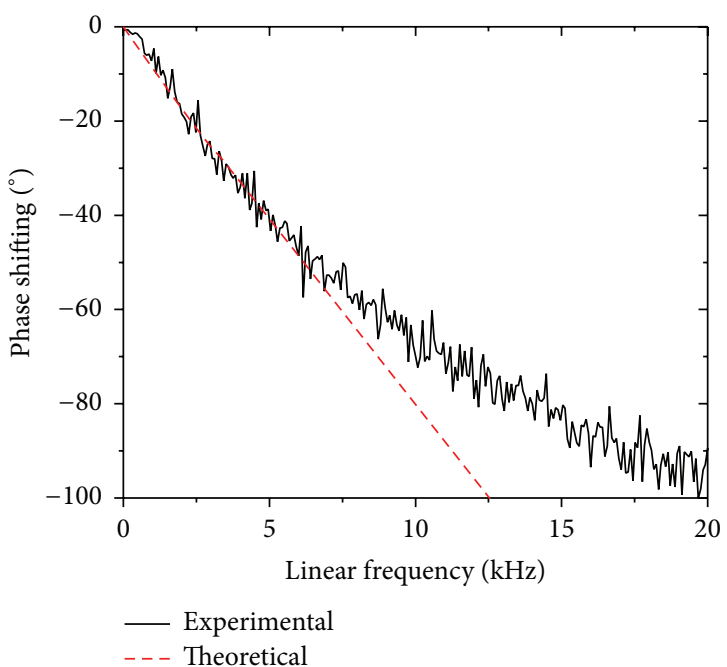

FIGURE 10: Theoretical and experimental phase shifting of AOM.

\section{Competing Interests}

The authors declare that they have no competing interests.

\section{Acknowledgments}

Thanks are due to Vicerrectoría de Investigación y Estudios de Posgrado (VIEP) of BUAP, Consejo Nacional de Ciencia y Tecnología (CONACyT), and Secretaría de Educación Pública (SEP) for their partial support to this project.

\section{References}

[1] P. St. J. Russell, "Photonic crystal fibres," Science, vol. 299, no. 5605, pp. 358-362, 2003.
[2] B. J. Eggleton, C. Kerbage, P. S. Westbrook, R. S. Windeler, and A. Hale, "Microstructured optical fiber devices," Optics Express, vol. 9, no. 13, pp. 698-713, 2001.

[3] O. Frazão, J. L. Santos, F. M. Araújo, and L. A. Ferreira, "Optical sensing with photonic crystal fibers," Laser and Photonics Reviews, vol. 2, no. 6, pp. 449-459, 2008.

[4] X. Y. Dong, H. Y. Tam, and P. Shum, “Temperature-insensitive strain sensor with polarization-maintaining photonic crystal fiber based Sagnac interferometer," Applied Physics Letters, vol. 90, no. 15, Article ID 151113, 3 pages, 2007.

[5] M. L. V. Tse, H. Y. Tam, L. B. Fu et al., "Fusion splicing holey fibers and single-mode fibers: a simple method to reduce loss and increase strength," IEEE Photonics Technology Letters, vol. 21, no. 3, pp. 164-166, 2009.

[6] H. Y. Fu, H. Y. Tam, L.-Y. Shao et al., "Pressure sensor realized with polarization-maintaining photonic crystal fiberbased Sagnac interferometer," Applied Optics, vol. 47, no. 15, pp. 2835-2839, 2008.

[7] G. Kim, T. Cho, K. Hwang et al., "Strain and temperature sensitivities of an elliptical hollow-core photonic bandgap fiber based on Sagnac interferometer," Optics Express, vol. 17, no. 4, pp. 2481-2486, 2009.

[8] E. Molina-Flores and E. Kuzin, "Thermically tuned erbiumdoped fiber ring laser," in Proceedings of the Conference on Lasers and Electro Optics/Quantum Electronics and Laser Science of Europe, Technical Digest, p. 620, Physical Society of Europe, June 2003.

[9] E. Molina-Flores and B. A. Ramírez-Solís, “Técnica de medición interferométrica espectral de la birrefringencia en fibras de cristal fotónico," Internet Electronic Journal. Nanociencia et Moletrónica, vol. 11, no. 1, pp. 2057-2064, 2013, http://www.revista-nanociencia.ece.buap.mx/11nr1/Rev.\%207Esteban\%20.pdf. 


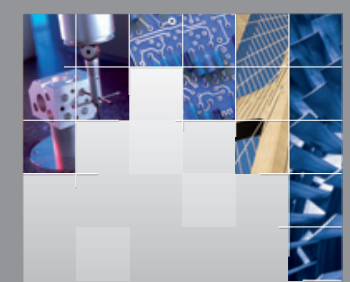

\section{Enfincering}
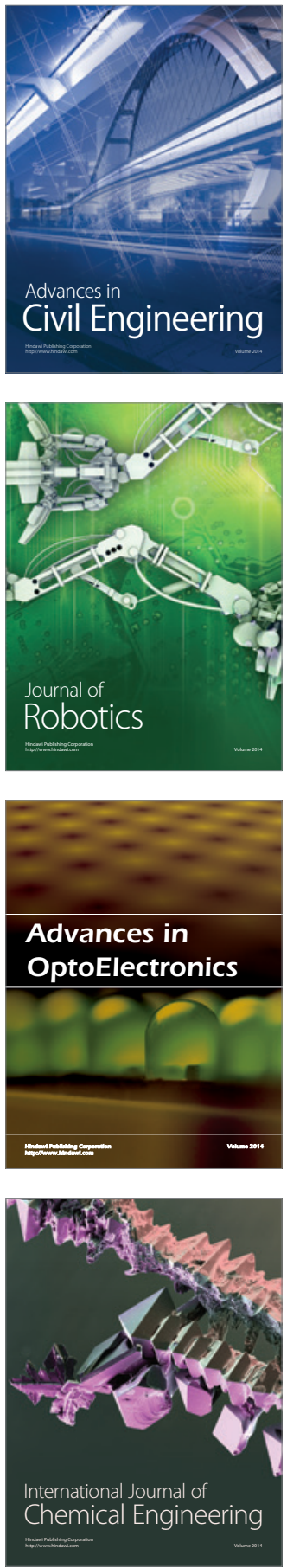

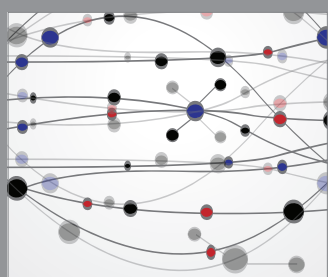

The Scientific World Journal

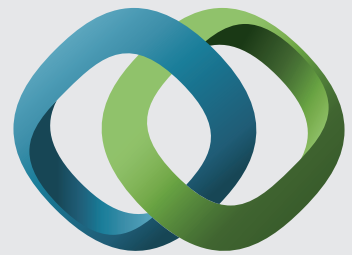

\section{Hindawi}

Submit your manuscripts at

http://www.hindawi.com
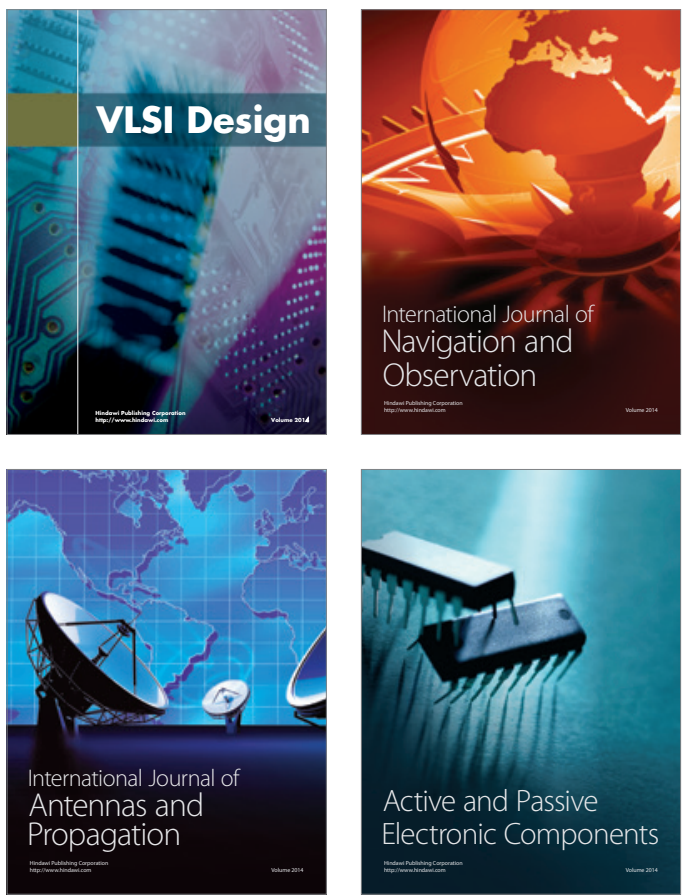
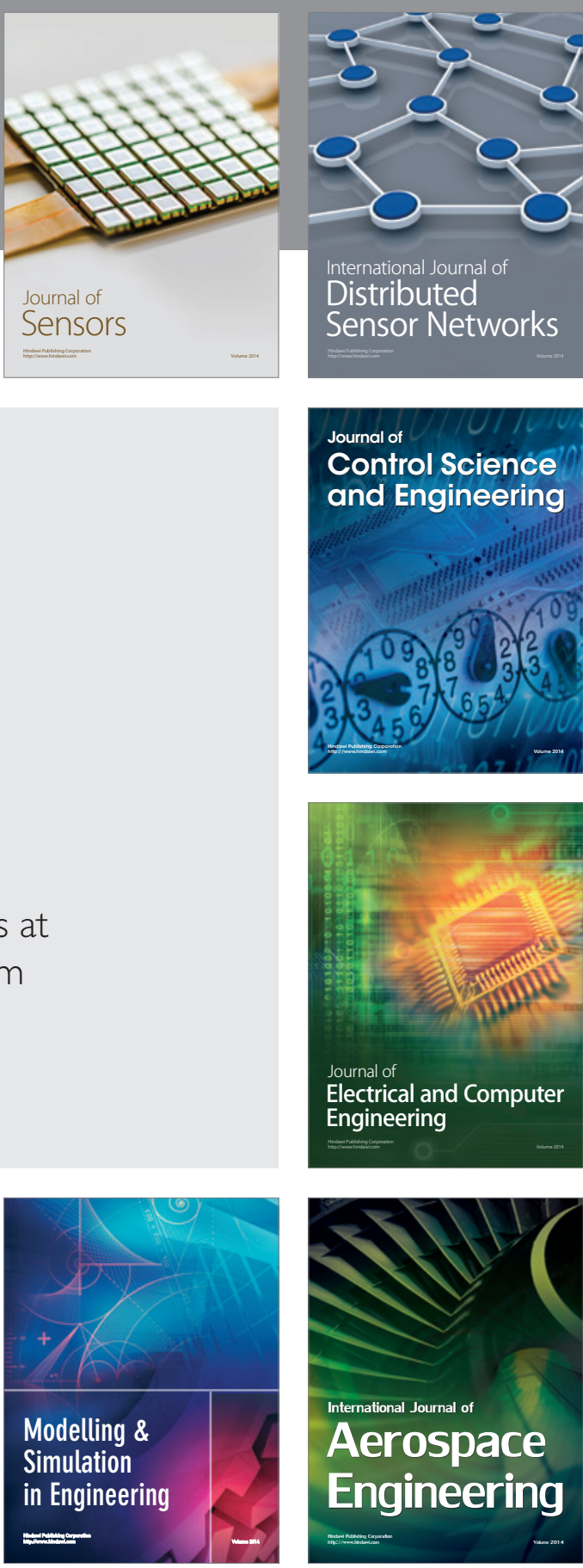

International Journal of

Distributed

Sensor Networks

Journal of

Control Science

and Engineering
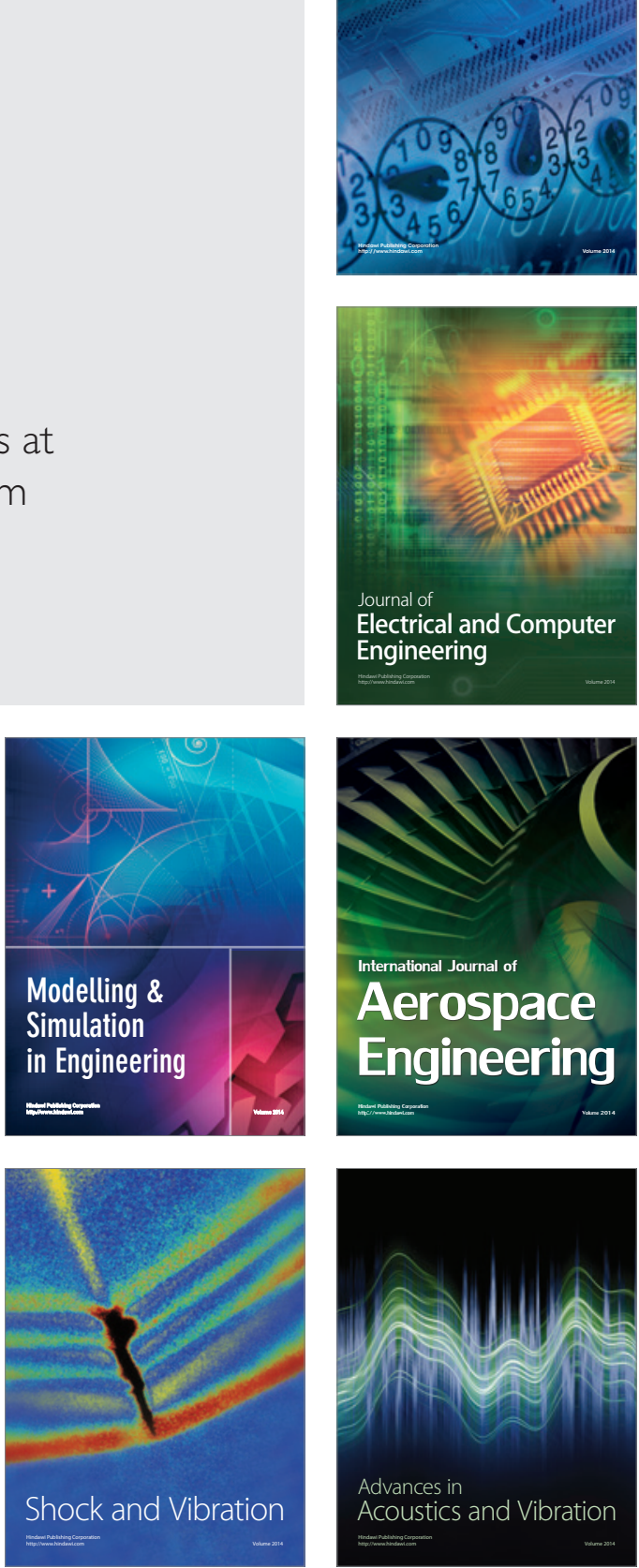